\title{
Deciphering the Network Effects of Deep Brain Stimulation in Parkinson's Disease
}

\author{
Gabriel Gonzalez-Escamilla (D) · Nabin Koirala • Manuel Bange • \\ Martin Glaser · Bogdan Pintea · Christian Dresel • Günther Deuschl • \\ Muthuraman Muthuraman · Sergiu Groppa
}

Received: August 5, 2021 / Accepted: December 21, 2021 / Published online: January 9, 2022

(C) The Author(s) 2022

\section{ABSTRACT}

Introduction: Deep brain stimulation of the subthalamic nucleus (STN-DBS) is an established therapy for Parkinson's disease (PD). However, a more detailed characterization of the targeted network and its grey matter (GM)

Muthuraman Muthuraman and Sergiu Groppa contributed equally to this work.

G. Gonzalez-Escamilla $(\bowtie) \cdot$ N. Koirala · M. Bange .

C. Dresel · M. Muthuraman · S. Groppa ( $\square)$

Movement Disorders and Neurostimulation,

Department of Neurology, Focus Program

Translational Neuroscience (FTN), Rhine Main

Neuroscience Network (rmn2), University Medical

Center of the Johannes Gutenberg University

Mainz, Langenbeckstrasse 1, 55131 Mainz, Germany

e-mail: ggonzale@uni-mainz.de

S. Groppa

e-mail: segroppa@uni-mainz.de

M. Glaser

Department of Neurosurgery, University Medical Center of the Johannes Gutenberg University

Mainz, Langenbeckstrasse 1, 55131 Mainz, Germany

B. Pintea

Department of Neurosurgery, University Hospital Bergmannsheil, Bürkle de la Camp-Platz 1, 44789 Bochum, Germany

\section{G. Deuschl}

Department of Neurology, Schleswig-Holstein University Hospital UKSH, Arnold-Heller-Straße 3, 24105 Kiel, Germany terminals that drive the clinical outcome is needed. In this direction, the use of MRI after DBS surgery is now possible due to recent advances in hardware, opening a window for the clarification of the association between the affected tissue, including white matter fiber pathways and modulated GM regions, and the DBS-related clinical outcome. Therefore, we present a computational framework for reconstruction of targeted networks on postoperative MRI.

Methods: We used a combination of preoperative whole-brain T1-weighted (T1w) and diffusion-weighted MRI data for morphometric integrity assessment and postoperative $\mathrm{T} 1 \mathrm{w}$ MRI for electrode reconstruction and network reconstruction in 15 idiopathic PD patients. Within this framework, we made use of DBS lead artifact intensity profiles on postoperative MRI to determine DBS locations used as seeds for probabilistic tractography to cortical and subcortical targets within the motor circuitry. Lastly, we evaluated the relationship between brain microstructural characteristics of DBStargeted brain network terminals and postoperative clinical outcomes.

Results: The proposed framework showed robust performance for identifying the DBS electrode positions. Connectivity profiles between the primary motor cortex (M1), supplementary motor area (SMA), and DBS locations were strongly associated with the stimulation intensity needed for the optimal 
clinical outcome. Local diffusion properties of the modulated pathways were related to DBS outcomes. STN-DBS motor symptom improvement was highly associated with cortical thickness in the middle frontal and superior frontal cortices, but not with subcortical volumetry.

Conclusion: These data suggest that STN-DBS outcomes largely rely on the modulatory interference from cortical areas, particularly M1 and SMA, to DBS locations.

Keywords: Deep brain stimulation; Parkinson's disease; Probabilistic tractography; Cortical thickness; Motor circuit

\section{Key Summary Points}

Despite advances in deep brain stimulation (DBS) technology that currently facilitate postoperative MRI acquisition, it is still not widely used, and its utility for studying widespread modulatory effects of DBS remains to be elucidated at the individual level.

We present a novel method to model DBS locations on postoperative MRI.

The hyperdirect pathway likely underlies the effects of DBS of the subthalamic nucleus (STN-DBS) in Parkinson's disease.

Optimal STN-DBS outcomes depend on the connectivity and the morphometric integrity of the modulated sensorimotor circuits.

\section{INTRODUCTION}

Deep brain stimulation of the subthalamic nucleus (STN-DBS) is an evidence-based and highly effective therapeutic option for patients with Parkinson's disease (PD) with motor fluctuations $[1,2]$. The efficacy of DBS stimulation for the improvement of major clinical motor symptoms like tremor, rigidity, and bradykinesia is indisputable. Nevertheless, despite its wide use and the clinical success of DBS [3, 4], its mechanism at the stimulated site and the anatomical structures influenced have not yet been fully elucidated [5]. Such information is however essential for modeling clinical dynamics or managing stimulation-related side effects. From the existing explanations about DBS operation, the now prevailing theories point to a stimulation-induced modulation of the pathological brain circuitry $[6,7]$. Therefore, we hypothesize that DBS not only alters the neural activity in the stimulated nuclei, but also influences the fiber tracts entering, exiting, or passing the stimulation site within the corticobasal ganglia-thalamocortical motor circuits. Among these circuits, the hyperdirect cortical STN projections, shown in studies on primates and recent studies on humans [8-10], presumably mediate the effects of STN-DBS $[11,12]$ through rapid movement inhibition [13]. This is further supported by electrophysiological reports of more prominent beta oscillatory activity in the basal ganglia than in motor-thalamic neurons, thus suggesting that the thalamus is more closely involved in tremor production [14] and is related to non-motor features in PD [15]. However, the exact characterization of the relationship between anatomical STN projections with stimulation parameters and disease-relevant clinical outcomes still remains elusive. Here, noninvasive evaluation of connecting white matter (WM) fiber tracts that are in direct contact with the DBS leads, based on diffusion-weighted magnetic resonance imaging (DWI), is key to advancing individualized DBS planning and therapy [16]. In this direction, even though a considerable amount of work has been conducted regarding the identification of WM pathways from DBS electrode locations and DBS-modulated gray matter (GM) regions as well as their relationship to clinical outcomes [17-20], previous work has lacked a detailed examination of the microstructural properties of the reconstructed pathways and the integrity of their connected GM terminals as derived from available morphometric and diffusion magnetic resonance imaging (MRI) metrics.

An important aspect of the variance in individual patient outcomes after STN-DBS is the 
variability in electrical stimulation parameters, which are chosen based on empirical, clinical observations regarding the exact DBS electrode contact positions. Appropriate modeling of DBS electrode contact locations is key for studying the effects of STN-DBS mechanisms. The volume of tissue activated (VTA) is a popular computational model for identifying the location of neural activation during DBS stimulation $[6,10,21]$. This method is limited and biased by assumptions about WM fiber orientation that influence axonal excitability [22]. These models may also rely on the quality of the DWI data and the tract reconstruction algorithm, and the limited predictability of active DBS contact locations [23]. Hence, there is a need for further methodological development for improved modeling of DBS targeted sites and effects.

In this study, we propose a novel methodological approach to integrate postoperative MRI for determining the DBS contact locations and analyze the WM pathways connecting the modeled electrode locations to nodes of the motor network in an individualized fashion using diffusion tensor imaging (DTI) and probabilistic tractography. We provide an example for the ability to detect the DBS electrode tip across different DBS systems. Further, we probe the method performance retrospectively in a cohort of PD patients. We evaluate the microstructural characteristics of the WM connections from the modeled stimulation sites to cortical targets and basal ganglia within the motor network, and test the relationship to the clinical outcomes and stimulation parameters of STN-DBS. The motivation for additionally studying the structural integrity of subcortical GM regions in our analysis was based on previous studies showing basal ganglia involvement, particularly the caudate and putamen, as well as the thalamus $[24,25]$, together with the existence of bilateral projections from the premotor and motor cortex to basal ganglia structures. We hypothesized (1) a relationship between DBS electrode locations and specific motor-related areas in the brain networks, and (2) that the location of these network areas would correlate with beneficial clinical outcomes. Additionally, we evaluated whether the local WM tissue microstructure of the active electrode contact or the GM characteristics at connected areas were associated with clinical outcomes after STNDBS.

\section{METHODS}

Fifteen patients with idiopathic PD without dementia selected for DBS treatment (11 male, mean age $63.3 \pm 8.2$, Hoehn and Yahr stage $(\mathrm{H} \& \mathrm{Y}) 3.5 \pm 0.8)$ were included in this study. Detailed demographic and clinical data are given in Table 1. Unified Parkinson's Disease Rating Scale part 3 (UPDRS-III) values in the medication OFF and stimulation ON condition were used to calculate the quotient to the preoperative UPDRS-III score in medication OFF. This quotient (further referred to as qUPDRS) was selected as the value for clinical outcome. Further, UPDRS-III lateralized hemi-body scores were computed by totaling the right and left appendicular UPDRS-III items (22-26) individually [26].

This study was approved by the institutional review board of the Medical Faculty of Schleswig-Holstein University Hospital UKSH, Kiel, Germany (approval number: AZ A 153/01). Each participant gave written informed consent before participation, and all the work described has been carried out in accordance with the principles expressed in the Declaration of Helsinki.

\section{Surgical Procedure and Stimulation Parameters}

The surgical procedure has been previously described in detail $[27,28]$. In brief, DBS electrodes (model 3389 DBS, Medtronic) were implanted bilaterally in the STN by stereotactic MRI, concomitant with microelectrode recording, ventriculography, or a combination of these techniques. The Medtronic 3389 lead consists of four cylindrical contacts, each $1.5 \mathrm{~mm}$ in height, separated by a $0.5 \mathrm{~mm}$ cylindrical insulator. Postoperatively, the optimal stimulation settings and antiparkinsonian medication were progressively adjusted according to the clinical response. The pulse setting 
Table 1 Cohort characteristics

\begin{tabular}{ll}
\hline Parkinson's disease patients $(\boldsymbol{n}=\mathbf{1 5})$ & No, $\mathbf{\text { SD }}$ \\
\hline Sex (male/female) & $11 / 4$ \\
Age (years) & $63.3 \pm 8.2$ \\
Disease duration (years) & $13.6 \pm 6.5$ \\
Preoperative H\&Y & $3.8 \pm 0.8$ \\
Postoperative H\&Y & $2.3 \pm 0.6$ \\
Preoperative UPDRS III (Med OFF) & $34.5 \pm 8.4$ \\
Preoperative UPDRS III (Med ON) & $17.4 \pm 9.0$ \\
Postoperative UPDRS III (Stimulation ON Med OFF) & $13.9 \pm 6$ \\
Postoperative UPDRS III (Stimulation ON Med ON) & $11.6 \pm 5.7$ \\
Preoperative dose of levodopa (mg/day) & $827 \pm 397.0$ \\
Postoperative dose of levodopa (mg/day) & $335 \pm 202.6$ \\
\hline
\end{tabular}

Demographic characteristics and clinical parameters assessed before and after STN-DBS surgery

Med medication, UPDRS-III Unified Parkinson's Disease Rating Scale, H\&Y Hoehn and Yahr scale

was $60 \mu \mathrm{s}$ in duration at $130 \mathrm{~Hz}$, with voltage adjusted to the individual patient. For our analysis, we considered the stimulation parameters at a stable state at 3 months after implantation. Clinicians who were blinded to the hypothesis and goals of this study performed the stimulation adjustment. We picked up the stimulation intensity at the active electrode as expressed in volts and considered it for further analyses. The medical treatment was individualized after DBS according to the clinical indication.

\section{MRI Data Acquisition}

All patients underwent preoperative high-resolution brain imaging using a $3 \mathrm{~T}$ MRI scanner (Philips Achieva, Philips Medical Systems, Best, Netherlands) with an eight-channel SENSE head coil. Whole-brain T1-weighted structural images were acquired using a standard magnetization-prepared rapid gradient-echo (MPRAGE) sequence $\quad(\mathrm{TR}=7.7 \mathrm{~ms}, \quad \mathrm{TE}=3.6 \mathrm{~ms}, \quad$ flip angle $=8^{\circ}, 160$ contiguous sagittal slices with $1 \mathrm{~mm}$ isometric voxel, field of view $=240 \times 240 \mathrm{~mm}$ ). DWI at $2 \mathrm{~mm}$ isometric voxel resolution covering a field of view of
$224 \times 224 \mathrm{~mm}$ was obtained using 32 gradient directions (b value $1000 \mathrm{~s} / \mathrm{mm}^{2}, \mathrm{TE}=59 \mathrm{~ms}$, $\mathrm{TR}=11,855 \mathrm{~ms}$, fat saturation "on," 60 contiguous slices).

On the first day after STN-DBS implantation, a whole-brain T1-weighted structural image was acquired using a standard MPRAGE sequence $\left(\mathrm{TR}=10.7 \mathrm{~ms}, \mathrm{TE}=1.96 \mathrm{~ms}\right.$, flip angle $=8^{\circ}, 160$ contiguous coronal slices with $2 \mathrm{~mm}$ isometric voxel size, field of view $=256 \times 256$ ) on a $1.5 \mathrm{~T}$ MRI scanner (Philips Achieva, Philips Medical Systems, Best, Netherlands). Further low-resolution fast fluid-attenuated inversion-recovery (FLAIR), and DWI sequences were recorded to exclude postoperative complications. All patients tolerated the MRI scans without side effects.

\section{Determination of Contact Position and Contact-Specific Masks}

Figure 1 depicts example cases of three patients implanted with electrodes from different companies currently manufacturing DBS systems, namely Medtronic DBS systems (Medtronic, Dublin, Ireland), Boston Scientific (Boston Scientific Corporation, Marlborough, MA, USA), 


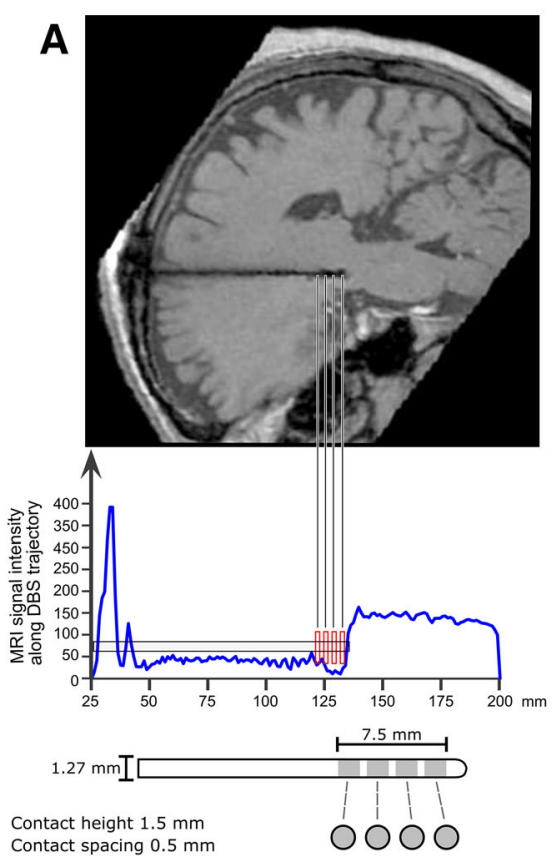

Fig. 1 Exemplary depiction of electrode positions and trajectory calculations for three different DBS electrode systems: a Medtronic 3389, b Boston Scientific Vercise, and $\mathbf{c}$ St. Jude L6170. From top to bottom: Depiction of the DBS lead artifact on postoperative T1 images. For each DBS electrode, the intensity profile was calculated along the lead artifact (upper row), and the electrode contact mask was determined by positioning the four contacts

and St. Jude Medical (Abbott Laboratories, Abbott Park, IL, USA). The contact positions of the implanted electrodes were modeled using an optimized lead position model, based on the postoperative T1-intensity profile following a two-step method. While the algorithm can generally be applied to leads from different vendors (as shown in Fig. 1), patients included in our analysis were exclusively implanted with Medtronic DBS electrodes.

Step 1. Delineation of contact positions: The lead was mathematically modeled by a straight line and the position was determined from a set of manually placed 3D space points (markers on the postoperative T1) along the electrode trajectory. The electrode trajectory was determined within the MRI signal intensity using all three orthogonal views (sagittal, coronal, and transverse). Markers were placed at the target points: near the points of exit and DBS lead dip, approximately linearly along the trajectory artifact (Fig. 1 a-c, upper panels). Finally, a three-dimensional least-square optimization procedure was used to determine the exact position of the trajectory, implemented in $\mathrm{R}$ (https://www.R-project.org/). Based on this optimized lead position, the intensity profile along the lead trajectory was extracted from the T1-weighted images (Fig. 1a-c, middle panel). The electrode contact positions were then determined at the center of the intensity dip apparent in the extracted intensity profile (Fig. 1a-c, lower panel).

Step 2. Active electrode contact stimulation volume modeling: The determined electrode contact positions were used to create masks based on spatial Gaussian weighting, centered at the exact contact positions (according to the manufacturer's annotations for the implanted DBS electrodes) and extended in two main 
directions: (i) along the lead dip (i.e., transverse plane of the MRI), to model contact dimensions, known from the manufacturer's annotations; and (ii) stimulation depth, covering the orthogonal directions to the electrode implantation (i.e., coronal and sagittal planes). See Fig. 2 for a detailed depiction of the Gaussian mask creation. The mask extension was restricted to a Gaussian shape with two standard deviations along the lead and two standard deviations in stimulation depth, resulting in isometric masks with $4.7 \mathrm{~mm}$ full width at half maximum (FWHM), corresponding to a radius of ca. $2.35 \mathrm{~mm}$. These parameters were selected considering existing literature reporting that neural elements up to a distance of $2 \mathrm{~mm}$ from the active contact might be excited by DBS [29-31]. The generated Gaussian masks were then used as seed masks in further probabilistic tractography analysis.

\section{MRI Processing}

T1-weighted structural images were used to extract measures of morphometric integrity, including cortical thickness values and subcortical volumes. The individual cortical surfaces were reconstructed from the skullstripped and intensity-inhomogeneity-corrected T1-weighted images using a semiautomatic pipeline implemented in FreeSurfer (http://surfer.nmr.mgh.harvard.edu), as described elsewhere [32]. Then, cortical thickness, defined as the distance between the gray/white and gray/CSF surface models, was calculated for motor regions: precentral, postcentral, middle frontal and superior frontal, and paracentral cortices, as defined in the Desikan-Killiany atlas [33]. Further, the FMRIB Software Library (FSL) FIRST tool was used to obtain subcortical volumes, as described previously [34]. As for cortical regions, we focused our subcortical analysis on regions of the motor system: thalamus, caudate nucleus, putamen, and globus pallidus externus (GPe) and internus (GPi).

\section{Diffusion Imaging Processing}

Diffusion imaging allows the study of brain microstructural characteristics based on the three-dimensional diffusion properties of water within the brain. Diffusion metrics include axial

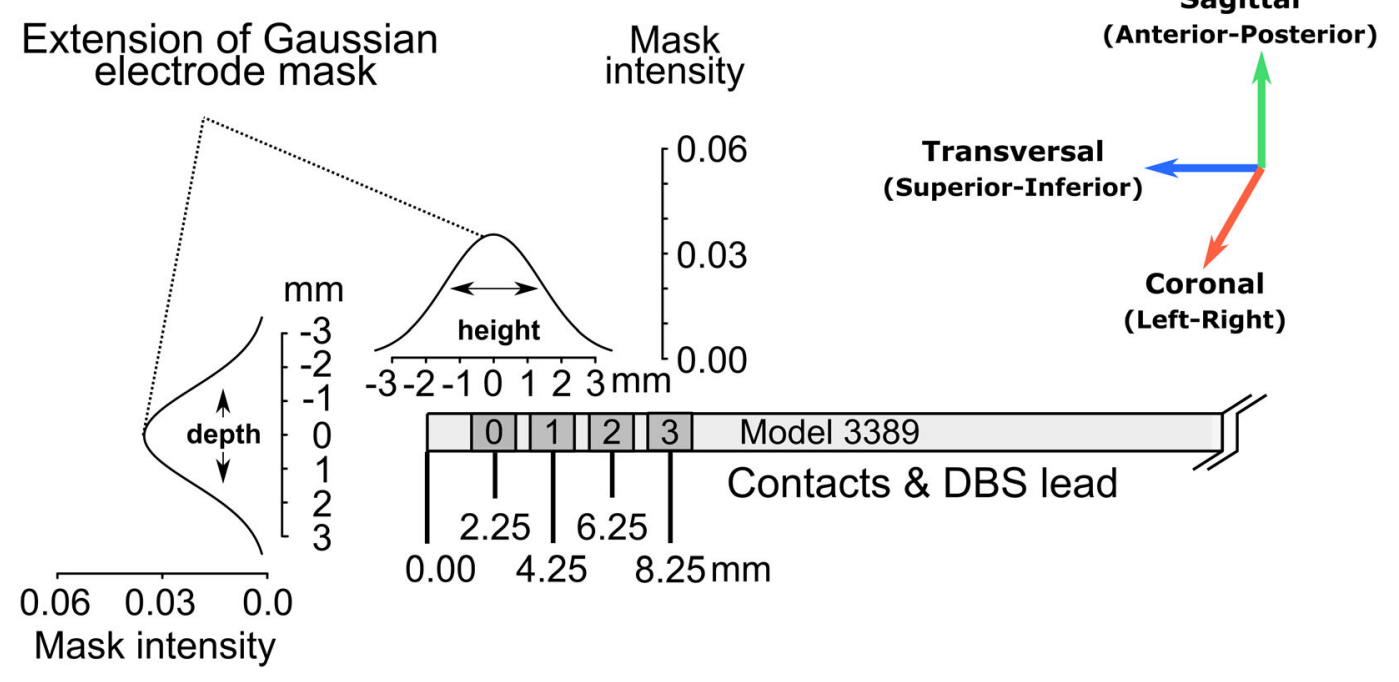

Fig. 2 Depiction of Gaussian masks. Exemplary illustration of the creation of the Gaussian masks at the distal end of the DBS electrode lead at contact locations. According to the MRI system coordinates, masks extended along the lead dip (height; transverse) and to the orthogonal directions (depth; sagittal and coronal) of a particular contact, in this example contact 0 . Mask intensity follows a Gaussian distribution from the center of the contact to the edges of the mask 
diffusivity (AD), radial diffusivity (RD), mean diffusivity (MD), and fractional anisotropy (FA), which were calculated using the FSL Diffusion Toolbox (FDT) (http://www.fmrib.ox.ac.uk/fsl/) [35]. Briefly, correction for eddy currents and head motion was first performed, followed by skull-stripping. Then, geometric distortions in the diffusion data were corrected by applying a non-rigid registration between the undistorted T1-weighted anatomical image and the first image (i.e., echo planar imaging [EPI] image with no diffusion weighting or b0 image) [36], and the transformation was finally applied to all volumes of the DWI images. After preprocessing, DTI was applied. Here, the six diffusion non-collinear dimensions were reduced via matrix diagonalization to its principal direction, resulting in three eigenvalues $(\lambda)$ at each voxel. $A D$ is the first eigenvalue and $R D$ is the mean of the second and third eigenvalues. $\mathrm{MD}$ is the mean of the three eigenvalues and FA is a nonlinear mapping of $\mathrm{RD}$ and $\mathrm{AD}$. FA is a summary measure of the microstructure including myelin axonal loss and increased extracellular space [37]. RD is a putative specific marker of myelin and $\mathrm{AD}$ of axonal damage [38]. MD reflects the membrane density and fluid viscosity [39]. All diffusivity metric images were aligned and affine-transformed into the Montreal Neurological Institute (MNI) space.

\section{Diffusion Probabilistic Tractography}

Three-dimensional representations of WM pathways, the so-called WM tractography, were obtained by firstly estimating the distribution of diffusion parameters with BEDPOSTX (Bayesian Estimation of Diffusion Parameters obtained using sampling techniques) [40], followed by a multi-fiber model allowing for tracing of fibers through regions of crossing or complexity $[40,41]$ as implemented in FSL [42]. Probabilistic tractography analysis is divided into three main stages, namely seeding, propagation, and termination. Seeding consists of defining the points from which the fiber bundles will be drawn-in our study, the Gaussian weighting masks of the contact positions. The propagation process consisted of gradually generating fibers by accounting for the uncertainty of the estimation. This results in probability maps representing the likelihood of a voxel forming part of a fiber and provides the multiple possible fiber directions emanating from the seed. Here, we drew 5000 streamline samples from our seed voxels to form an estimate of the probability distribution of connections from each seed voxel. The last step, termination of the fiber tracking, aims at avoiding propagating the fibers to voxels where robustness of the diffusion tensor vector field is not assured. This step was based on well-defined termination criteria, including a minimum FA threshold (0.2) and turning angle threshold $\left(80^{\circ}\right)$. Spurious connections were discarded by including only voxels through which at least $10 \%$ of all streamline samples had passed [43]. To allow bias-free definition of target termination regions, we built masks for cortical seeds from anatomical coordinates known from a meta-analysis for activation studies [44]. The generated target masks were spheres with a radius of $2 \mathrm{~mm}$ (and $3 \mathrm{~mm}$ for comparison) centered at the primary motor cortex (M1: -37 -21 58), premotor dorsal (PMd: $-30-458$ ), and premotor ventral (PMv: -50522 ; and supplementary motor area [SMA]: $-2-7$ 55) $[44,45]$. Coordinates were transformed into MNI space using GingerALE [46]. Target masks of the globus pallidus (internus and externus) were generated from the MNI probability atlas by including the entire areas [47].

A further tractography analysis aimed at generating voxel-based connectivity index maps (reflecting the abovementioned probability in connection of each voxel in the target from the seed) and delimiting the cortical connections from the contact positions to the connections of the corticospinal tract (CST). This tract also included the M1 mask but had a conjunction mask of the ipsilateral cerebral peduncle region and internal capsule [48]. This information was fed into further statistical analysis as connectivity values. To evaluate the link between connectivity patterns from resulting individual probabilistic tractography maps and DBS outcome, each voxel on the reconstructed tract and subject was assigned a value 
on the corresponding $\mathrm{FA}, \mathrm{AD}, \mathrm{RD}$, and $\mathrm{MD}$ maps.

\section{Statistical Analyses}

The statistical analyses were performed under the general linear model using SPSS software (version 16.0, SPSS Inc, Chicago, IL, USA) and MATLAB R2018b (The MathWorks Inc. Natick, MA, USA). Data from both hemispheres were pooled to improve the statistical power of the analysis, and one-sample $t$ tests were used to test whether the STN-DBS clinical outcome measurements (i.e. qUPDRS) deviated from a zero mean.

For extracted connectivity values for the probability maps, an analysis of variance (ANOVA) was conducted to evaluate its association with clinical outcomes, including connectivity values to target region as withinsubject factor (six levels: GPe, GPi, SMA, M1, PMd, PMv) and DBS stimulation intensity as dependent variable. A second ANOVA was modeled with the same within-subject factors but qUPDRS as dependent variable. Post hoc analyses were conducted, depicting specific associations between the dependent variables (DBS stimulation intensity or qUPDRS) and the connectivity values to each target independently (GPe, GPi, SMA, M1, PMd, PMv).

To analyze the association between diffusion parameters and clinical outcomes, we ran two independent ANOVAs, using either DBS stimulation intensity or qUPDRS as dependent variables and the diffusion parameters of the reconstructed CST (four levels: $\mathrm{FA}, \mathrm{MD}, \mathrm{AD}$, and $\mathrm{RD)}$ as within-subject factors.

To evaluate the association between cortical thickness and clinical outcomes, an ANOVA model was used. This included DBS stimulation intensity as dependent variable and cortical thickness (five levels: precentral, postcentral, middle frontal, superior frontal gyrus, paracentral lobule) as within-subject factors. A second ANOVA was modeled for qUPDRS as dependent variable.

For the subcortical volumetry, again ANOVAs were set to evaluate the association between DBS clinical outcomes and subcortical integrity. DBS stimulation intensity and
qUPDRS were used as continuous predictors. Both ANOVAs included the subcortical volumes (four levels: thalamus, caudate nucleus, putamen, and pallidum) as within-subject factors.

Correction for multiple comparisons was performed across ANOVA models using the false discovery rate (FDR), and the subsequent post hoc analyses were adjusted for multiple comparisons using the Bonferroni test at a 95\% confidence level.

\section{RESULTS}

\section{DTI Analysis and Diffusion Parameters}

The analysis performed to assess the WM microstructural changes using various diffusion parameters including $\mathrm{FA}, \mathrm{MD}, \mathrm{AD}, \mathrm{RD}$, and the probability of major (f1) and secondary (f2) fiber direction showed no significant associations for the effect of interest with respect to the stimulation intensity.

\section{Probabilistic Tractography}

Probabilistic tractography was performed from seed (Gaussian masks from the active electrode contact) to target (cortical-M1, SMA, PMd, $\mathrm{PMv}$, and subcortical-GPe, GPi) regions to assess the connectivity profile from the stimulation site to various cortical and subcortical regions. The ANOVA evidenced significant associations between DBS intensity and connectivity across seed regions $\left(F_{(5,140)}=2.35\right.$, $p<0.05)$. Specifically, DBS stimulation intensity was negatively associated with the connectivity of the SMA $(r=-0.43, F=6.26$, $p=0.018$, Fig. $3 \mathrm{~A})$ and $\mathrm{M} 1(r=-0.46, F=7.44$, $p=0.011$, Fig. 3B). The ANOVA including qUPDRS revealed significant associations with regional connectivity $\left(F_{(5,140)}=8.81, p<0.05\right)$, where associations between DBS stimulation intensity appeared with SMA $(r=-0.37$, $F=3.58, p=0.034)$ and $\mathrm{M} 1(r=-0.36, F=4.2$, $p=0.025)$. No associations with connectivity indices from the cortical (PMd, PMv) or subcortical (GPi or GPe) regions of interest were found. 
A

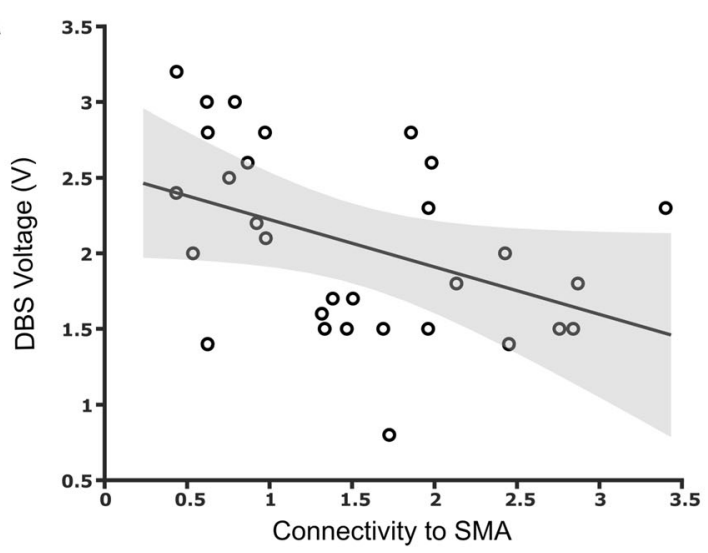

C

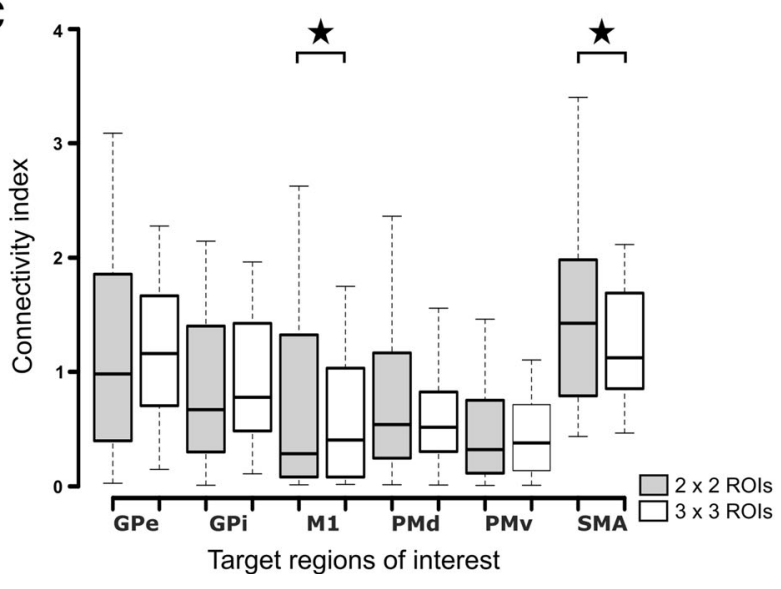

B
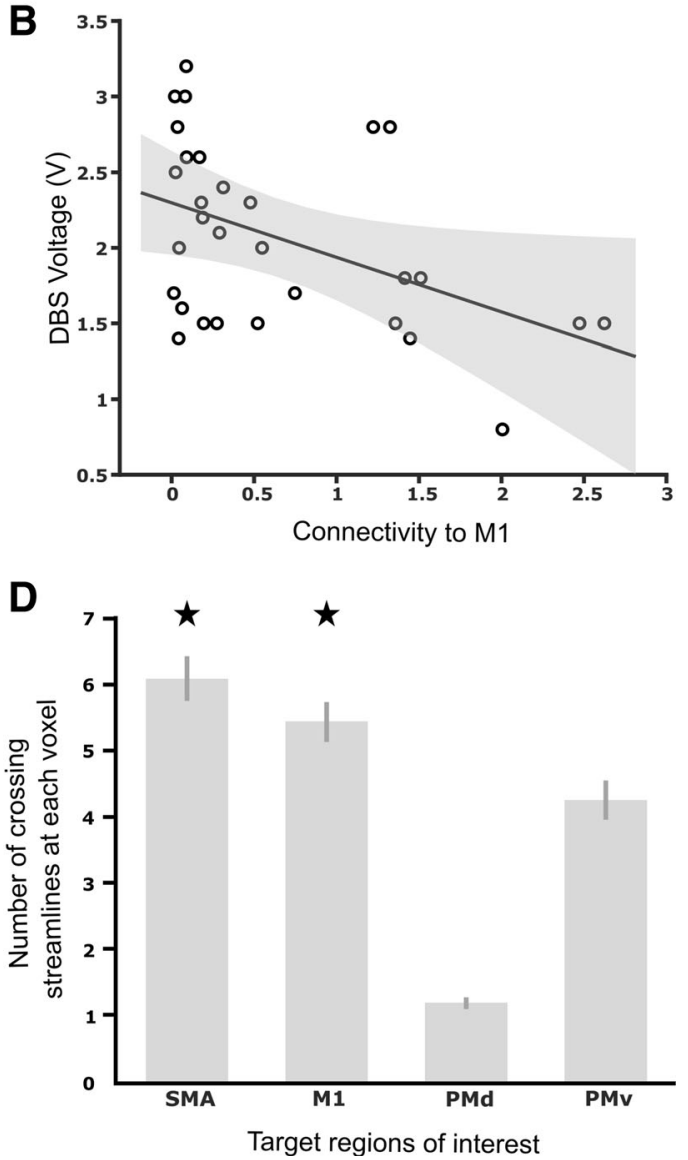

Fig. 3 Involvement of SMA and M1. Regression analysis between stimulation intensity at the active contact and the connectivity indices for a SMA $(r=0.43, F=6.26$, $p=0.018)$ and b M1 $(r=0.46, F=7.44, p=0.011)$, respectively. c Comparison of connectivity indices across target mask of two sizes centered at MNI coordinates from

Reconstructed WM pathways from the Gaussian masks showed a clear connectivity profile reaching cortical regions in the motor and frontal regions (Fig. 4B). Within this tract, an association was confirmed between qUPDRS $\left.\left(F_{(3,} 84\right)=2.95, \quad p<0.05\right)$ and the diffusion parameters. In particular, worse DBS outcomes were associated with higher $\mathrm{AD}(r=0.44$, $F=6.71, \quad p=0.015)$ and $\mathrm{RD} \quad(r=-0.29$, $F=4.47, p=0.04$ ) values (Fig. $4 \mathrm{C}$ ).

To shed further light on whether specific connections within the CST, particularly to M1 and SMA, may influence the observed connectivity, their overlap with DBS stimulation was plotted (Fig. 5). Visually, the lower stimulation a meta-analysis of activation studies (see "Methods" section for detailed information), evidencing effects of size only in M1 and SMA. d The mean number of reconstructed DTI streamlines crossing each voxel of the Gaussian seed masks and connecting to a cortical target region

intensities from DBS locations overlapped with both CST tracts (M1 and SMA), thus confirming the results between connectivity and voltage (Fig. 3a and b).

\section{Cortical and Subcortical Analysis}

Cortical thickness and subcortical volumes were further assessed to evaluate whether GM characteristics of the network regions are related to the DBS stimulation clinical outcomes. The fitted models revealed an association between qUPDRS and cortical thickness $\left(F_{(4,112)}=49.7\right.$, $p<0.01)$, in which the middle frontal cortex 

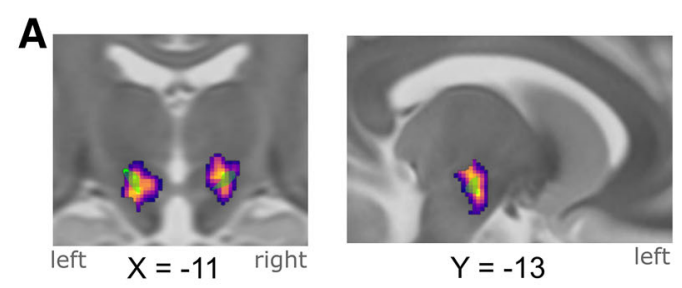

STN-motor

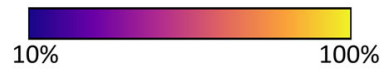

B

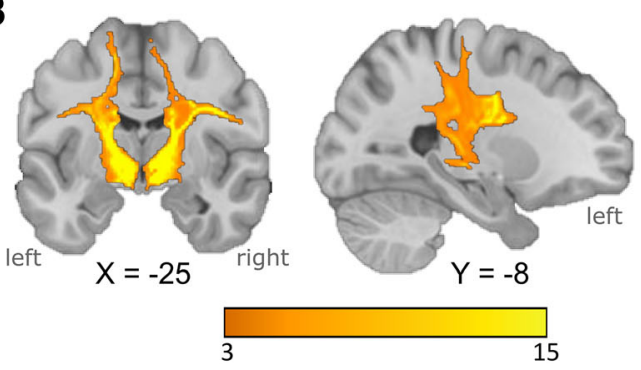

Fig. 4 Connectivity analysis among cortical masks. a Maximum probabilistic map depicting the individual locations of the binary Gaussian masks at the active electrode contact location across participants, where the color scale indicates none $(10 \%$, dark purple) or all patients $(100 \%$, yellow) having overlapping contact masks at each voxel. In green, the sensorimotor part of the STN as defined by Accolla et al. [81] is used as anatomical reference. b Reconstructed white matter pathways, as determined by probabilistic tractography, connecting the seed masks from DBS locations with the target cortical regions, while

( $r=0.49, F=8.92, p=0.006$, Fig. 6$)$ and superior frontal cortex $(r=0.38, F=4.80, p=0.037)$ were the only two significant variables. No further associations between qUPDRS and cortical thickness in the precentral, postcentral, or paracentral cortical thickness were found (all $p>0.1$ corrected for multiple comparisons).

Analyses on subcortical regions revealed no associations between structural integrity and stimulation intensity or qUPDRS after STN-DBS, for either qUPDRS $\left(F_{(3,84)}=1.29, p>0.1\right)$ or DBS stimulation intensity $\left(F_{(3,} 84\right)=1.66$, $p>0.1)$.

\section{DISCUSSION}

Using a novel method for modeling DBS leads and contact locations according to the artifact
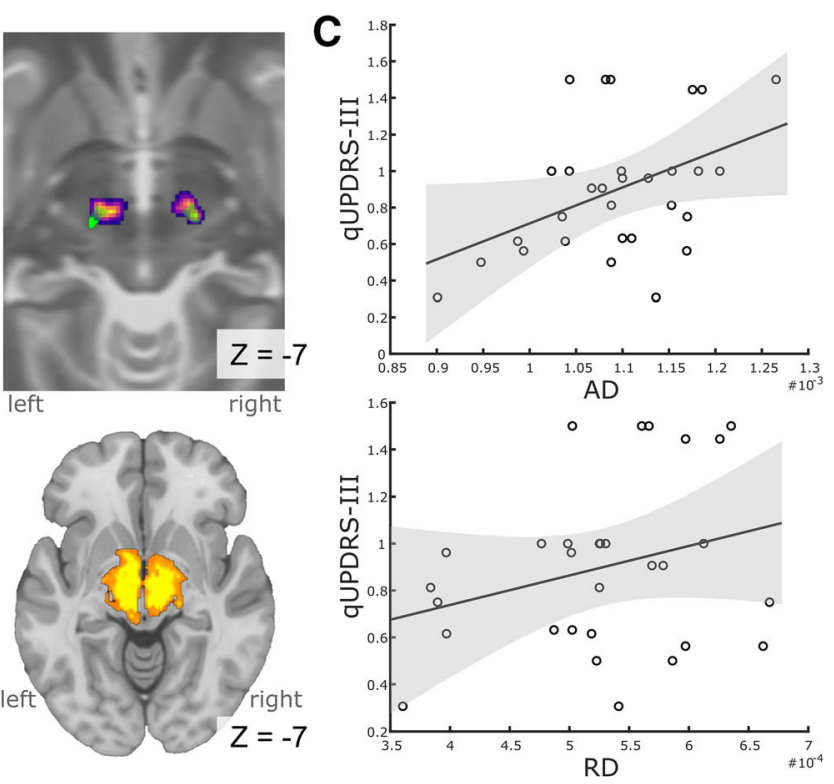

going through the basal ganglia and the internal capsule to the cortex. Warm color coding represents the number of DBS electrodes (in each brain hemisphere) that have the pathways crossing through. For example, dark orange areas depict pathways that were targeted by few electrodes, while brighter colors show that these voxels contain connections that were identified by a higher number of electrodes. c Regression plots depicting the association between diffusion metrics (axial diffusivity- $A D$, and radial diffusivity-RD) and qUPDRS-III

intensity spectrum on postoperative wholebrain T1-weighted images, we show that the postoperative outcome of STN-DBS is strongly associated with individual connectivity patterns derived from diffusion MRI probabilistic tractography. Particularly, we show that structural connectivity from active stimulation contacts to M1 and SMA, among studied cortical and subcortical regions, were the only regions strongly associated with the modulatory effects of STN-DBS. Considering cortical thickness as a parameter of GM microstructural tissue integrity $[49,50]$ and diffusion metrics of WM microstructure, we provide further evidence that the STN-DBS clinical outcome is strongly associated with the individual tissue characteristics and connectivity profiles extending from the local characteristics at the stimulation site 

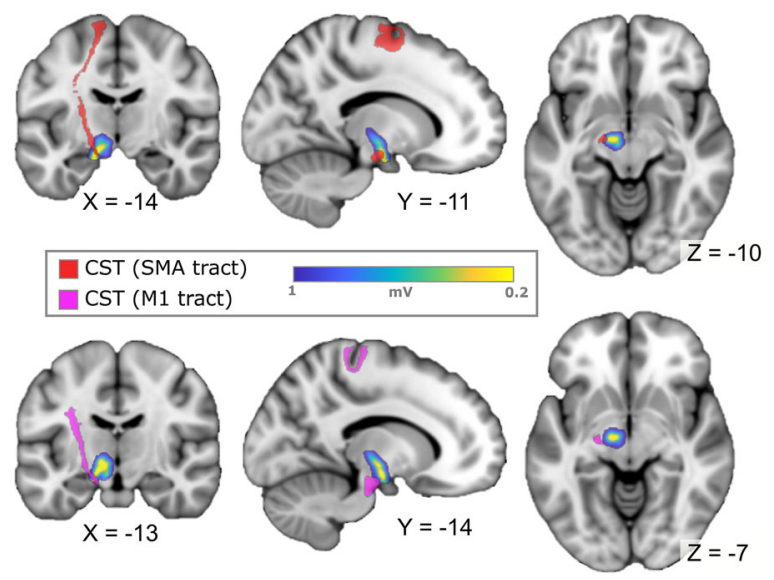

Fig. 5 Corticospinal tracts and DBS intensity. Representation of the sensorimotor corticospinal tracts (CST) in relation to stimulation at electrode locations. The color scale represents the mean applied stimulation intensity at the effective electrode locations among all patients. The CST part projecting to the SMA is represented in red, and projections to $\mathrm{M} 1$ are shown in violet. The tracts were constructed with either SMA or M1 masks as target regions, while tracts were seeded from the cerebral peduncle (CP) [82]. Tract masks are freely available at http://lrnlab.org/

or volumetric properties of the studied basal ganglia structures.

\section{WM Projections Between STN-DBS Contact Locations and Motor Regions Relate to Clinical Outcomes}

The proposed method for active DBS electrode contact modeling identifies the connectivity profile for defining effective DBS implantation and optimal outcome. We showed that connectivity from the stimulation site to cortical regions within the motor circuits is strongly associated with optimal clinical effects for motor symptoms and with the lowest stimulation current for optimal outcome during STNDBS. This is in accord with the recently proposed network mechanism of action, meaning a global modulation of distributed brain networks $[5-7,10]$. From all included regions within the motor circuits, only the connectivity to M1 and SMA was shown to be related to lower DBS intensities required for effective STN-DBS outcomes. Concordant with our results, atrophy in the SMA and premotor cortex has recently been reported as a predictor of DBS outcomes in PD [51]. SMA activation has been hypothesized to take place through activation of fibers within the hyperdirect pathway [18]. Abnormal connectivity between the STN and M1 and modulation of M1 excitability by STN-DBS have also been proposed as an important fingerprint of PD-related pathophysiological alterations $[52,53]$. Our results confirm the importance of the SMA and M1 in the response to STN-DBS.

The direct involvement of the M1 for the effects of the STN-DBS has also been suggested in rodent PD models [11, 12]. First, in animal models, DBS-induced antidromic spikes in layer $\mathrm{V}$ pyramidal cells triggered a dampened oscillation of local field potentials in the cortex with a resonant frequency around $120 \mathrm{~Hz}$ [12]. Second, using optogenetics and solid-state optics, direct activation of cortical afferents from M1 projecting to the STN region was explicitly associated with therapeutic benefit [11]. Of
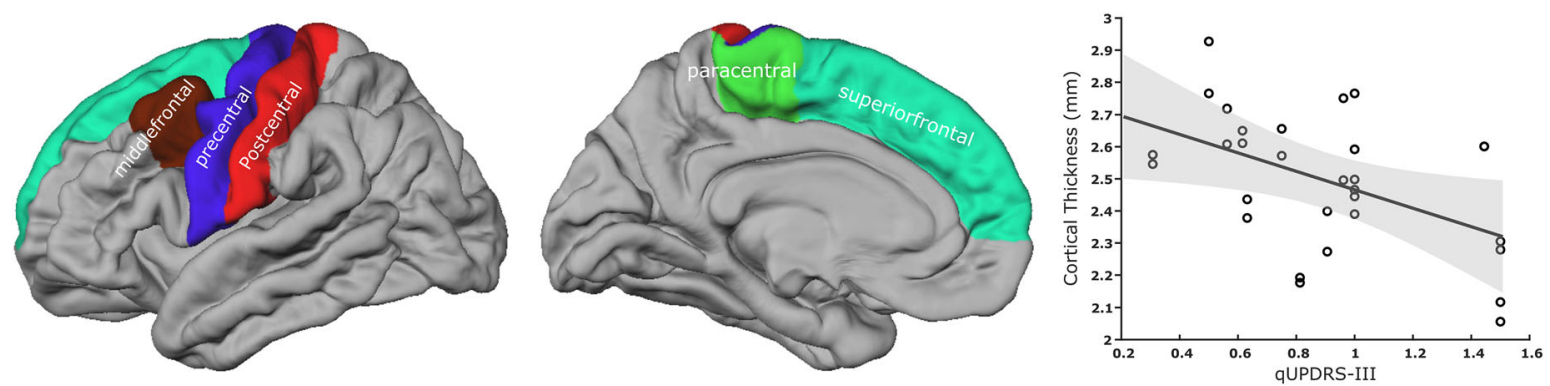

Fig. 6 Cortical thickness analysis. Regions of interest used for cortical thickness association analyses $(r=0.49$, $F=8.92, p=0.006$ ) between the morphometric integrity

of the middle frontal cortex and the quotient of post- to preoperative UPDRS scores (qUPDRS) 
note, direct stimulation of the pyramidal tract would drive the activation of cranial or spinal motor neurons, leading to muscle contractions $[54,55]$, which were not observed in any of our patients. Based on these studies, the current results suggest that STN-DBS may modulate the connections between M1 and STN and SMA and STN via the hyperdirect pathway and functionally related circuits, in a way that the corticobasal-ganglia-cortical loop is normalized towards the level of healthy circuits. Therefore, in patients with PD, STN-DBS might achieve maximum clinical outcome improvement through modulation of these pathways [10].

The association between correct DBS contact implantation and beneficial outcomes may be directly linked to the fact that DBS-programmed electrical stimulation parameters restrain the extent of the applied electrical field and thus, to some extent, the type of neural elements that are activated and inhibited [56]. This association can currently be extended beyond the local properties of the targeted region to include specific fibers to be implicated in the improvement of particular symptoms. For example, when studying the relationship between electrode connectivity, derived from diffusion MRI data, and improvement in cardinal disease symptoms, the connectivity from active DBS electrode locations to M1 explained improvement in tremor, connectivity to SMA explained improvement in bradykinesia, and connectivity to both the SMA and prefrontal regions explained improvements in rigidity [10].

The duality of DBS as a research and clinical tool with which we can investigate the physiological underpinnings of brain dysfunction associated with a pathological condition and modulate brain circuitry as a therapeutic intervention can be translated into clinical settings to deliver individualized treatments and diminish potential side effects. For example, the neurologist could be informed about the connectivity profile of STN-DBS contact locations to adjust the parameters and maximize the outcome effects.

\section{WM Connectivity Microstructure and DBS Outcomes}

Measures of WM microstructure, particularly $\mathrm{AD}$ and $\mathrm{RD}$, and their alterations in localized brain regions have previously shed light on important processes of PD pathology [57-59], due to their ability to depict even small variations in structural connectivity $[60,61]$. The finding that $\mathrm{AD}$ and $\mathrm{RD}$ are related to the qUPDRS-III substantiated that both the integrity of the axons and the level of myelination within the targeted network influence the outcome of STN-DBS. This strengthens our hypothesis that the STN-DBS effect is relayed on the network pathways from the active DBS electrode contacts.

\section{Cortical-Subcortical Morphometry and STN-DBS Clinical Outcome}

For our analyses we used the quotient of preoperative and postoperative scores as clinical outcome of STN-DBS. This approach permits a reduction in the inter-individual variations and therefore an unbiased estimation of associations with GM circuit integrity [51]. In the current study, the clinical motor improvement after STN-DBS correlated with cortical thickness values in the middle frontal and superior frontal cortices but not in the precentral, postcentral, or paracentral cortices, suggesting a topographic specificity of the STN-DBS effect. In this sense, patients with atrophy in the frontal cortices before surgery had a clinically inferior STNDBS outcome relative to those with preserved cortical morphology. Previous studies have suggested that subthalamic activity is modulated by cortical activity in the frontal regions, particularly in the alpha $(7-13 \mathrm{~Hz})$ and beta $(15-35 \mathrm{~Hz})$ bands [62]. Moreover, in PD patients, cortical atrophy in the frontal cortex and the other regions used in the current study is accelerated already in the early stages of disease [25], and is also associated with the clinical status of motor symptoms [63], PD degeneration [64, 65], disease progression [66], nonmotor symptoms such as cognitive impairment [67], and DBS outcomes [51]. Concordantly, it 
was more recently shown that the clinical effectiveness of STN-DBS is strongly associated with the WM network connectivity profile of the frontal, prefrontal, and cingulate cortices [68]. Our data take these findings a step further, into a new framework that stresses not only the role of the microstructural integrity WM pathways but also the anatomical integrity of the regions belonging to the motor network for optimal improvement after STN-DBS.

Therefore, this complementary data on disease-relevant subcortical regions rounds out our proposed framework, allowing for more indepth hypothesis testing. The lack of associations between subcortical structures and the clinical outcome or stimulation parameters can be explained by the fact that the distribution of regional atrophy predominantly involves distributed brain regions at once, and individual evaluation of each region may reflect relatively narrow inter-subject variability, since all the included patients were at similar disease stages (indicated by the H\&Y scores), with similar disease duration and motor symptoms before STN-DBS therapy. These results uncover a highly specific relationship between STN-DBS effects and the structural integrity of regions within the motor circuit and directly connected to the stimulation sites.

\section{Study Limitations}

This study does have some limitations. First, although we provide an example for the electrode detection on postoperative MRI for different DBS systems, the analyses were performed using DBS electrodes from a single vendor. Whereas validation of detection performance with electrodes from different vendors with increased sample sizes would be desirable, the effects and possible bias of combining patients with different DBS systems still needs further clarification, while usage of multicentric data to increase the sample size could add a confounding effect related to differences in surgical procedures that cannot be easily accounted for. Also, relatedly, for segmented leads, allowing for directional stimulation, identification of the rotation along the insertion axis would need to be taken into account. Even when work has been conducted in this direction [69-71], no current perfect (automatic) algorithm for this aim exists, which is partly due to the symmetry of the observed artifact and the suboptimal selection of the artifact center on the postsurgical images. A further limitation is related to MRI acquisitions, which intrinsically restrict the discrimination of subcortical brain structures and precise delimitation of electrode boundaries at current common magnetic fields (1.5 and 3 Tesla). While delimitation of smaller brain structures is currently enabled by the use of brain atlases, DBS technology still needs further improvement before being able to introduce ultra-high-field MRI sequences for detailed electrode localization. This is not only for patient safety during the acquisition of postoperative MRI, but also to avoid signal distortions that may appear in MR images due to the material used for constructing DBS electrodes. Moreover, there are some effects that may have remained hidden due to the modest sample size, including the link between cortical integrity and WM connectivity to the STN.

A further possible shortcoming of our study if the fact that targets for tractography were defined as spheres, which are relatively small compared with the size of the cortical areas of interest. The primary motivation in using this approach was to provide a bias-free definition of target termination regions-i.e., different parcellation schemes deliver different organization and anatomical delimitations, with a direct impact on connectivity measurements [72]. Secondly, by controlling the size across the brain regions, we avoid the bias wherein larger regions get a larger number of terminating streamlines $[72,73]$, thus increasing the comparability of the connectivity index across regions while facilitating its interpretability.

It should be noted that different algorithms already exist for electrode localization and reconstruction, as well as for the modeling of the stimulation volumes (see e.g. [6, 74-77]). Unfortunately, a direct comparison among software is not straightforward due to differences in the algorithms implemented and the manual refinements often required. A 
distinctive feature of the framework presented herein is the ability to work directly with postoperative MRI data for electrode localization, which circumvents the effects of interpolation during image preprocessing as in other pipelines.

Of note, the present results go beyond the utilization of a novel framework for evaluating DBS outcomes and its imaging underpinnings, to largely validate recent reported findings in predicting DBS outcomes from imaging features $[6,10,25,51,78,79]$, recently reviewed in Gonzalez-Escamilla et al. [80]. This is of high relevance in the face of the current replication crisis affecting many scientific studies.

\section{CONCLUSIONS}

Altogether, our data suggest that modeling of DBS electrode contacts based on postoperative MRI acquisitions could be used as a tool to investigate DBS effects. The current analyses revealed that the clinical effects of STN-DBS in PD distinctively relate to the connectivity profile from the stimulation location within the STN to M1 and SMA regions, and the morphometric integrity of these connected regions. Structural MRI and DTI pattern studies are of high importance for refining STN-DBS targeting and potentially improving the network modulation achieved, with clinical implications. The developed framework can be applied to refine DBS targeting and improve the analyses of targeted neuromodulation of specific networks through modeling of the systemic effects achieved at an individualized MRI-driven level.

\section{ACKNOWLEDGEMENTS}

We thank Rosalind Gilchrist for proofreading the manuscript. We thank the participants of the study.

Funding. No funding or sponsorship was received for this study or publication of this article. The Rapid Service Fee was funded by the authors.
Authorship. All named authors meet the International Committee of Medical Journal Editors (ICMJE) criteria for authorship for this article, take responsibility for the integrity of the work as a whole, and have given their approval for this version to be published.

Author Contributions. Gabriel GonzalezEscamilla drafted the manuscript, analyzed the data, and prepared the figures. Nabin Koirala and Manuel Bange contributed to acquisition of data. Bogdan Pintea contributed to analysis of data. Martin Glaser, Christian Dresel, Günther Deuschl, and Muthuraman Muthuraman contributed to data interpretation and manuscript revision. Sergiu Groppa contributed to conception and design of the study. Gabriel GonzalezEscamilla and Sergiu Groppa were co-corresponding authors.

Disclosures. Gabriel Gonzalez-Escamilla has nothing to disclose. Nabin Koirala has nothing to disclose. Manuel Bange has nothing to disclose. Martin Glaser has nothing to disclose. Bogdan Pintea has nothing to disclose. Christian Dresel has nothing to disclose. Günther Deuschl has nothing to disclose. Muthuraman Muthuraman has nothing to disclose. Sergiu Groppa has nothing to disclose.

Compliance with Ethics Guidelines. This study was approved by the institutional review board of the Medical Faculty of Schleswig-Holstein University Hospital UKSH, Kiel, Germany (Approval number: AZ A 153/01). Each participant gave written informed consent before participation and all the work described has been carried out in accordance to the principles expressed in the Declaration of Helsinki.

Data Availability. The data that support the findings of this study are available on request from the corresponding author. The data are not publicly available due to institutional restrictions. All data was processed using standard pipelines as described in manuscript text. Further code would be made available upon reasonable request to the corresponding author. 
Open Access. This article is licensed under a Creative Commons Attribution-NonCommercial 4.0 International License, which permits any non-commercial use, sharing, adaptation, distribution and reproduction in any medium or format, as long as you give appropriate credit to the original author(s) and the source, provide a link to the Creative Commons licence, and indicate if changes were made. The images or other third party material in this article are included in the article's Creative Commons licence, unless indicated otherwise in a credit line to the material. If material is not included in the article's Creative Commons licence and your intended use is not permitted by statutory regulation or exceeds the permitted use, you will need to obtain permission directly from the copyright holder. To view a copy of this licence, visit http://creativecommons.org/licenses/by$\mathrm{nc} / 4.0 /$.

\section{REFERENCES}

1. Odekerken VJ, van Laar T, Staal MJ, et al. Subthalamic nucleus versus globus pallidus bilateral deep brain stimulation for advanced Parkinson's disease (NSTAPS study): a randomised controlled trial. Lancet Neurol. 2013;12:37-44. https://doi.org/10. 1016/S1474-4422(12)70264-8.

2. Deuschl G, Paschen S, Witt K. Clinical outcome of deep brain stimulation for Parkinson's disease. Handb Clin Neurol. 2013;116:107-28.

3. Fasano A, Appel-Cresswell S, Jog M, et al. Medical management of Parkinson's disease after initiation of deep brain stimulation. Can J Neurol Sci. 2016;43:626-34. https://doi.org/10.1017/cjn.2016. 274.

4. Rowland NC, Sammartino F, Lozano AM. Advances in surgery for movement disorders. Mov Disord. 2017;32:5-10. https://doi.org/10.1002/mds.26636.

5. Muthuraman M, Koirala N, Ciolac D, et al. Deep brain stimulation and L-DOPA therapy: concepts of action and clinical applications in Parkinson's disease. Front Neurol 2018; (In Press).

6. Horn A, Wenzel G, Irmen F, et al. Deep brain stimulation induced normalization of the human functional connectome in Parkinson's disease. Brain. 2019. https://doi.org/10.1093/brain/awz239.
7. Lozano AM, Lipsman N, Bergman H, et al. Deep brain stimulation: current challenges and future directions. Nat Rev Neurol. 2019;15:148-60. https://doi.org/10.1038/s41582-018-0128-2.

8. Brunenberg EJ, Moeskops P, Backes WH, et al. Structural and resting state functional connectivity of the subthalamic nucleus: identification of motor STN parts and the hyperdirect pathway. PLoS ONE. 2012;7:e39061. https://doi.org/10.1371/journal. pone.0039061.

9. Nambu A, Tokuno H, Takada M. Functional significance of the cortico-subthalamo-pallidal [ hyperdirect'pathway. Neurosci Res. 2002;43:111-7.

10. Akram H, Sotiropoulos SN, Jbabdi S, et al. Subthalamic deep brain stimulation sweet spots and hyperdirect cortical connectivity in Parkinson's disease. Neuroimage. 2017;158:332-45. https://doi. org/10.1016/j.neuroimage.2017.07.012.

11. Gradinaru V, Mogri M, Thompson KR, et al. Optical deconstruction of parkinsonian neural circuitry. Science. 2009;324:354-9. https://doi.org/10.1126/ science. 1167093.

12. Li Q, Ke Y, Chan DC, et al. Therapeutic deep brain stimulation in Parkinsonian rats directly influences motor cortex. Neuron. 2012;76:1030-41. https:// doi.org/10.1016/j.neuron.2012.09.032.

13. Chen W, de Hemptinne C, Miller AM, et al. Prefrontal-subthalamic hyperdirect pathway modulates movement inhibition in humans. Neuron. 2020;106:579-588.e573. https://doi.org/10.1016/j. neuron.2020.02.012.

14. Du G, Zhuang P, Hallett M, et al. Properties of oscillatory neuronal activity in the basal ganglia and thalamus in patients with Parkinson's disease. Transl Neurodegener. 2018;7:17. https://doi.org/10. 1186/s40035-018-0123-y.

15. Niccolini F, Wilson H, Giordano B, et al. Sleep disturbances and gastrointestinal dysfunction are associated with thalamic atrophy in Parkinson's disease. BMC Neurosci. 2019;20:55. https://doi.org/ 10.1186/s12868-019-0537-1.

16. O'Halloran RL, Chartrain AG, Rasouli JJ, et al. Case study of image-guided deep brain stimulation: magnetic resonance imaging-based white matter tractography shows differences in responders and nonresponders. World Neurosurg. 2016. https:// doi.org/10.1016/j.wneu.2016.08.103.

17. Vanegas-Arroyave N, Lauro PM, Huang L, et al. Tractography patterns of subthalamic nucleus deep brain stimulation. Brain. 2016;139:1200-10. https://doi.org/10.1093/brain/aww020. 
18. Accolla EA, Herrojo Ruiz M, Horn A, et al. Brain networks modulated by subthalamic nucleus deep brain stimulation. Brain. 2016;139:2503-15. https://doi.org/10.1093/brain/aww182.

19. Koirala N, Fleischer V, Granert O, et al. Network effects and pathways in Deep brain stimulation in Parkinson's disease. Annu Int Conf IEEE Eng Med Biol Soc. 2016;2016:5533-6. https://doi.org/10. 1109/EMBC.2016.7591980.

20. Lin $\mathrm{H}, \mathrm{Na} \mathrm{P}$, Zhang $\mathrm{D}$, et al. Brain connectivity markers for the identification of effective contacts in subthalamic nucleus deep brain stimulation. Hum Brain Mapp. 2020;41:2028-36. https://doi. org/10.1002/hbm.24927.

21. Mahlknecht P, Akram H, Georgiev D, et al. Pyramidal tract activation due to subthalamic deep brain stimulation in Parkinson's disease. Mov Disord. 2017;32:1174-82. https://doi.org/10.1002/ mds. 27042 .

22. Duffley G, Anderson DN, Vorwerk J, et al. Evaluation of methodologies for computing the deep brain stimulation volume of tissue activated. J Neural Eng. 2019. https://doi.org/10.1088/17412552/ab3c95.

23. Reich MM, Horn A, Lange F, et al. Probabilistic mapping of the antidystonic effect of pallidal neurostimulation: a multicentre imaging study. Brain. 2019;142:1386-98. https://doi.org/10.1093/brain/ awz046.

24. Burton EJ, McKeith IG, Burn DJ, et al. Cerebral atrophy in Parkinson's disease with and without dementia: a comparison with Alzheimer's disease, dementia with Lewy bodies and controls. Brain. 2004;127:791-800. https://doi.org/10.1093/brain/ awh088.

25. Tessa C, Lucetti C, Giannelli M, et al. Progression of brain atrophy in the early stages of Parkinson's disease: a longitudinal tensor-based morphometry study in de novo patients without cognitive impairment. Hum Brain Mapp. 2014;35:3932-44. https://doi.org/10.1002/hbm.22449.

26. Purger D, Gibson EM, Monje M. Myelin plasticity in the central nervous system. Neuropharmacology. 2016;110:563-73. https://doi.org/10.1016/j. neuropharm.2015.08.001.

27. Herzog J, Hamel W, Wenzelburger R, et al. Kinematic analysis of thalamic versus subthalamic neurostimulation in postural and intention tremor. Brain. 2007;130:1608-25.

28. Groppa S, Herzog J, Falk D, et al. Physiological and anatomical decomposition of subthalamic neurostimulation effects in essential tremor. Brain.
2014;137:109-21. https://doi.org/10.1093/brain/ awt304.

29. Ranck JB. Which elements are excited in electrical stimulation of mammalian central nervous system: a review. Brain Res. 1975;98:417-40.

30. Butson CR, Maks CB, McIntyre CC. Sources and effects of electrode impedance during deep brain stimulation. Clin Neurophysiol. 2006;117:447-54. https://doi.org/10.1016/j.clinph.2005.10.007.

31. Gunalan K, Howell B, McIntyre CC. Quantifying axonal responses in patient-specific models of subthalamic deep brain stimulation. Neuroimage. 2018;172:263-77. https://doi.org/10.1016/j. neuroimage.2018.01.015.

32. Fischl B. FreeSurfer. Neuroimage. 2012;62:774-81. https://doi.org/10.1016/j.neuroimage.2012.01.021.

33. Desikan RS, Segonne F, Fischl B, et al. An automated labeling system for subdividing the human cerebral cortex on MRI scans into gyral based regions of interest. Neuroimage. 2006;31:968-80. https://doi. org/10.1016/j.neuroimage.2006.01.021.

34. Patenaude B, Smith SM, Kennedy DN, et al. A Bayesian model of shape and appearance for subcortical brain segmentation. Neuroimage. 2011;56:907-22. https://doi.org/10.1016/j. neuroimage.2011.02.046.

35. Jenkinson M, Beckmann CF, Behrens TE, et al. FSL. Neuroimage. 2012;62:782-90. https://doi.org/10. 1016/j.neuroimage.2011.09.015.

36. Bhushan C, Haldar JP, Choi S, et al. Co-registration and distortion correction of diffusion and anatomical images based on inverse contrast normalization. Neuroimage. 2015;115:269-80. https://doi. org/10.1016/j.neuroimage.2015.03.050.

37. Song S-K, Sun S-W, Ju W-K, et al. Diffusion tensor imaging detects and differentiates axon and myelin degeneration in mouse optic nerve after retinal ischemia. Neuroimage. 2003;20:1714-22.

38. Harsan LA, Poulet P, Guignard B, et al. Brain dysmyelination and recovery assessment by noninvasive in vivo diffusion tensor magnetic resonance imaging. J Neurosci Res. 2006;83:392-402. https:// doi.org/10.1002/jnr.20742.

39. Alexander AL, Hurley SA, Samsonov AA, et al. Characterization of cerebral white matter properties using quantitative magnetic resonance imaging stains. Brain Connect. 2011;1:423-46.

40. Behrens TE, Berg HJ, Jbabdi S, et al. Probabilistic diffusion tractography with multiple fibre orientations: what can we gain? Neuroimage. 2007;34: 
144-55. https://doi.org/10.1016/j.neuroimage. 2006.09.018.

41. Behrens TEJ, Woolrich MW, Jenkinson M, et al. Characterization and propagation of uncertainty in diffusion-weighted MR imaging. Magn Reson Med. 2003;50:1077-88. https://doi.org/10.1002/mrm. 10609 .

42. Smith SM, Jenkinson $M$, Johansen-Berg $H$, et al. Tract-based spatial statistics: voxelwise analysis of multi-subject diffusion data. Neuroimage. 2006;31: 1487-505.

43. Boorman ED, O'Shea J, Sebastian C, et al. Individual differences in white-matter microstructure reflect variation in functional connectivity during choice. Curr Biol. 2007;17:1426-31.

44. Mayka MA, Corcos DM, Leurgans SE, et al. Threedimensional locations and boundaries of motor and premotor cortices as defined by functional brain imaging: a meta-analysis. Neuroimage. 2006;31:1453-74.

45. Argyelan $M$, Carbon $M$, Niethammer $M$, et al. Cerebellothalamocortical connectivity regulates penetrance in dystonia. J Neurosci. 2009;29: 9740-7.

46. Eickhoff SB, Laird AR, Grefkes C, et al. Coordinatebased ALE meta-analysis of neuroimaging data: a random-effects approach based on empirical estimates of spatial uncertainty. Hum Brain Mapp. 2009;30:2907.

47. Mazziotta J, Toga A, Evans A, et al. A probabilistic atlas of the human brain: theory and rationale for its development the international consortium for brain mapping (ICBM). Neuroimage. 1995;2: 89-101.

48. Jbabdi S, Woolrich MW, Andersson JLR, et al. A Bayesian framework for global tractography. Neuroimage. 2007;37:116-29.

49. Tamnes CK, Ostby Y, Fjell AM, et al. Brain maturation in adolescence and young adulthood: regional age-related changes in cortical thickness and white matter volume and microstructure. Cereb Cortex. 2010;20:534-48. https://doi.org/10.1093/ cercor/bhp118.

50. Hoagey DA, Rieck JR, Rodrigue KM, et al. Joint contributions of cortical morphometry and white matter microstructure in healthy brain aging: a partial least squares correlation analysis. Hum Brain Mapp. 2019;40:5315-29. https://doi.org/10.1002/ hbm. 24774 .

51. Muthuraman M, Deuschl G, Koirala N, et al. Effects of DBS in parkinsonian patients depend on the structural integrity of frontal cortex. Sci Rep. 2017;7:43571. https://doi.org/10.1038/srep43571.

52. Baudrexel S, Witte T, Seifried C, et al. Resting state fMRI reveals increased subthalamic nucleus-motor cortex connectivity in Parkinson's disease. Neuroimage. 2011;55:1728-38. https://doi.org/10. 1016/j.neuroimage.2011.01.017.

53. Hanajima R, Ashby P, Lozano AM, et al. Single pulse stimulation of the human subthalamic nucleus facilitates the motor cortex at short intervals. J Neurophysiol. 2004;92:1937-43.

54. Tommasi G, Krack P, Fraix V, et al. Pyramidal tract side effects induced by deep brain stimulation of the subthalamic nucleus. J Neurol Neurosurg Psychiatry. 2008;79:813-9. https://doi.org/10.1136/ jnnp.2007.117507.

55. Xu W, Miocinovic S, Zhang J, et al. Dissociation of motor symptoms during deep brain stimulation of the subthalamic nucleus in the region of the internal capsule. Exp Neurol. 2011;228:294-7. https://doi.org/10.1016/j.expneurol.2010.08.007.

56. Herrington TM, Cheng JJ, Eskandar EN. Mechanisms of deep brain stimulation. J Neurophysiol. 2016;115:19-38. https://doi.org/10.1152/jn.00281. 2015.

57. Kamagata K, Hatano T, Okuzumi A, et al. Neurite orientation dispersion and density imaging in the substantia nigra in idiopathic Parkinson disease. Eur Radiol. 2016;26:2567-77. https://doi.org/10. 1007/s00330-015-4066-8.

58. Safai A, Prasad S, Chougule T, et al. Microstructural abnormalities of substantia nigra in Parkinson's disease: a neuromelanin sensitive MRI atlas based study. Hum Brain Mapp. 2020;41:1323-33. https:// doi.org/10.1002/hbm.24878.

59. Zhang Y, Wu IW, Tosun D, et al. Progression of regional microstructural degeneration in Parkinson's disease: a multicenter diffusion tensor imaging study. PLoS ONE. 2016;11: e0165540. https:// doi.org/10.1371/journal.pone.0165540.

60. Fjell AM, Sneve MH, Grydeland H, et al. Relationship between structural and functional connectivity change across the adult lifespan: a longitudinal investigation. Hum Brain Mapp. 2017;38:561-73. https://doi.org/10.1002/hbm.23403.

61. Soares JM, Marques P, Alves V, et al. A hitchhiker's guide to diffusion tensor imaging. Front Neurosci. 2013;7:31. https://doi.org/10.3389/fnins.2013. 00031.

62. Litvak V, Jha A, Eusebio A, et al. Resting oscillatory cortico-subthalamic connectivity in patients with 
Parkinson's disease. Brain. 2011;134:359-74. https://doi.org/10.1093/brain/awq332.

63. Kang D-z, Chen F-y, Wang F-y, et al. Brain gray matter volume changes associated with motor symptoms in patients with Parkinson's disease. Chin Neurosurg J. 2015;1:9.

64. Ibarretxe-Bilbao N, Junque C, Segura B, et al. Progression of cortical thinning in early Parkinson's disease. Mov Disord. 2012;27:1746-53. https://doi. org/10.1002/mds.25240.

65. Lyoo CH, Ryu YH, Lee MS. Topographical distribution of cerebral cortical thinning in patients with mild Parkinson's disease without dementia. Mov Disord. 2010;25:496-9.

66. Mak E, Su L, Williams GB, et al. Baseline and longitudinal grey matter changes in newly diagnosed Parkinson's disease: ICICLE-PD study. Brain. 2015;138:2974-86. https://doi.org/10.1093/brain/ awv211.

67. Hanganu A, Monchi O. Structural neuroimaging markers of cognitive decline in Parkinson's disease. Parkinson's Dis. 2016;2016:3217960. https://doi. org/10.1155/2016/3217960.

68. Koirala N, Fleischer V, Glaser M, et al. Frontal lobe connectivity and network community characteristics are associated with the outcome of subthalamic nucleus deep brain stimulation in patients with Parkinson's disease. Brain Topogr. 2018;31:311-21. https://doi.org/10.1007/s10548-017-0597-4.

69. Hellerbach A, Dembek TA, Hoevels M, et al. DiODe: directional orientation detection of segmented deep brain stimulation leads: a sequential algorithm based on CT imaging. Stereotact Funct Neurosurg. 2018;96:335-41. https://doi.org/10.1159/ 000494738.

70. Reinacher P, Krüger M, Coenen V, et al. Determining the orientation of directional deep brain stimulation electrodes using 3D rotational fluoroscopy. Am J Neuroradiol. 2017;38:1111-6.

71. Sitz A, Hoevels M, Hellerbach A, et al. Determining the orientation angle of directional leads for deep brain stimulation using computed tomography and digital x-ray imaging: a phantom study. Med Phys. 2017;44:4463-73. https://doi.org/10.1002/mp. 12424 .

72. Zalesky A, Fornito A, Harding IH, et al. Whole-brain anatomical networks: does the choice of nodes matter? Neuroimage. 2010;50:970-83. https://doi. org/10.1016/j.neuroimage.2009.12.027.
73. Girard G, Whittingstall K, Deriche R, et al. Towards quantitative connectivity analysis: reducing tractography biases. Neuroimage. 2014;98:266-78. https://doi.org/10.1016/j.neuroimage.2014.04.074.

74. D'Albis T, Haegelen C, Essert C, et al. PyDBS: an automated image processing workflow for deep brain stimulation surgery. Int $\mathrm{J}$ Comput Assist Radiol Surg. 2015;10:117-28. https://doi.org/10. 1007/s11548-014-1007-y.

75. Johansson JD, Alonso F and Wardell K. Patientspecific simulations of deep brain stimulation electric field with aid of in-house software ELMA. In: Annual International Conference of the IEEE Engineering in Medicine and Biology Society IEEE Engineering in Medicine and Biology Society Annual International Conference 2019; 2019: 5212-5216. https://doi.org/10.1109/EMBC.2019. 8856307

76. Noecker AM, Choi KS, Riva-Posse P, et al. StimVision software: examples and applications in subcallosal cingulate deep brain stimulation for depression. Neuromodulation. 2018;21:191-6. https://doi.org/10.1111/ner.12625.

77. Lauro PM, Vanegas-Arroyave N, Huang L, et al. DBSproc: an open source process for DBS electrode localization and tractographic analysis. Hum Brain Mapp. 2016;37:422-33. https://doi.org/10.1002/ hbm. 23039.

78. Horn A, Reich M, Vorwerk J, et al. Connectivity predicts deep brain stimulation outcome in Parkinson disease. Ann Neurol. 2017;82:67-78. https://doi.org/10.1002/ana.24974.

79. Wang Q, Akram H, Muthuraman M, et al. Normative vs patient-specific brain connectivity in deep brain stimulation. Neuroimage. 2021;224:117307. https://doi.org/10.1016/j.neuroimage.2020. 117307.

80. Gonzalez-Escamilla G, Muthuraman M, Ciolac D, et al. Neuroimaging and electrophysiology meet invasive neurostimulation for causal interrogations and modulations of brain states. Neuroimage. 2020;220: 117144. https://doi.org/10.1016/j. neuroimage.2020.117144.

81. Accolla EA, Dukart J, Helms G, et al. Brain tissue properties differentiate between motor and limbic basal ganglia circuits. Hum Brain Mapp. 2014;35: 5083-92. https://doi.org/10.1002/hbm.22533.

82. Archer DB, Vaillancourt DE, Coombes SA. A template and probabilistic atlas of the human sensorimotor tracts using diffusion MRI. Cereb Cortex. 2018;28:1685-99. https://doi.org/10.1093/cercor/ bhx066. 